\title{
The role of molecular breast imaging in predicting complete tumor response to treatment and residual tumor extent following neoadjuvant therapy
}

\author{
SUSANNA NUVOLI ${ }^{1}$, SARAH GALASSI $^{1}$, ILARIA GELO ${ }^{1}$, GAIA ROCCHITTA $^{2}$, ALESSANDRO FANCELLU ${ }^{3}$, \\ PIER ANDREA SERRA ${ }^{2}$, GIUSEPPE MADEDDU ${ }^{1}$ and ANGELA SPANU ${ }^{1}$ \\ Units of ${ }^{1}$ Nuclear Medicine, ${ }^{2}$ Pharmacology and ${ }^{3}$ Surgery, Department of Clinical and \\ Experimental Medicine, University of Sassari, I-07100 Sassari, Italy
}

Received October 28, 2017; Accepted March 1, 2018

DOI: $10.3892 /$ or.2018.6299

\begin{abstract}
The aim of the present study was to investigate the usefulness of molecular breast imaging (MBI) in predicting complete tumor response to treatment and residual tumor extent following neoadjuvant therapy. A consecutive series of 43 female patients with large or locally advanced primary breast cancer, scheduled for surgery following neoadjuvant therapy, was retrospectively reviewed. Prior to surgery, all patients underwent MBI using a high-resolution semiconductor-based device for image acquisition. MBI data were correlated with surgical histopathological findings. Spearman's correlation coefficient was calculated to assess differences in residual tumor size with MBI and histopathological examination. From the images obtained using MBI, 7 patients were negative for residual tumors with pathological complete response (specificity, 100\%) and positive in 34/36 patients with residual disease (sensitivity, 94.4\%), including 26/27 patients with unifocal and $8 / 9$ patients with multicentric/multifocal tumors, 5 of which exhibited multiple microscopic foci scattered in a fibrotic area. Overall accuracy was $95.3 \%$ and the positive predictive value (PPV) and negative predictive value (NPV) were 100 and $77.8 \%$, respectively. MBI was false-negative in one patient with a $2.5-\mathrm{cm}$ invasive ductal carcinoma located close to the chest wall and in one patient with microscopic foci of epithelial carcinoma. In the patients with unifocal residual tumors, correlation of tumor size between MBI and histopathology was $\mathrm{r}=0.981(\mathrm{P}<0.0001)$; however, MBI overestimated the number of lesions in one of these cases. In the patients with multifocal/multicentric tumors, MBI adequately assessed residual tumor extent in $5 / 8$ positive cases, overestimating
\end{abstract}

Correspondence to: Professor Angela Spanu, Unit of Nuclear Medicine, Department of Clinical and Experimental Medicine, University of Sassari, Viale San Pietro 8, I-07100 Sassari, Italy

E-mail: angela.spanu@email.it

Key words: breast cancer, neoadjuvant therapy, molecular breast imaging the number of lesions in one case and underestimating tumor extent in 2 further cases with microscopic foci scattered in a fibrotic area. MBI proved to be a highly accurate diagnostic tool in predicting complete tumor response to neoadjuvant therapy and residual tumor extent, correlating with surgical histopathological findings in $86 \%$ of overall cases. A positive result was always associated with the presence of residual disease and MBI tumor size was strongly correlated with histopathological analysis mainly in unifocal residual tumors.

\section{Introduction}

Neoadjuvant systemic therapy, either chemotherapy or anti-hormonal therapy, is increasingly used as a first-line therapeutic option in patients with locally advanced operable, primarily non-operable and inflammatory primary breast cancer since it may lead to a local downstage of the primary tumor, thus increasing the rate of conservative surgical treatments (1).

Neoadjuvant therapy may also sterilize sites of local and distant metastases and improve long-term and disease-free survival (2).

Moreover, it offers the possibility of assessing early in vivo tumor-response to systemic treatments, guiding the oncologist in selecting the most appropriate post-surgical therapies, since the degree of response to neoadjuvant therapy represents an important prognostic factor (3).

Therefore, it is crucial to have an imaging method able to predict and quantify tumor response to neoadjuvant therapy.

The response of the primary tumor to neoadjuvant treatment is generally assessed by both clinical examination and conventional imaging procedures, such as mammography and breast ultrasonography, which however rely only on changes in size or morphological characteristics, demonstrating inherent limitations in differentiating residual tumors from fibrotic replacement and over or underestimating residual disease extent (4).

Radioisotopic imaging of the breast (breast scintigraphy or scintimammography) with the technetium-labelled cationic lipophilic radiotracers sestamibi and tetrofosmin provides functional imaging, selectively identifying viable tumors 
and increasing the specificity of morphological imaging methods (5-7). Radiotracer uptake and accumulation in neoplastic cells indeed depend on vascular, biochemical and metabolic factors such as regional blood flow, angiogenesis, plasma and mitochondrial membrane potential as well as tissue metabolism (8-10).

However, scintimammography, when acquired with a conventional general-purpose gamma camera, is recognized to have a low sensitivity in the detection of primary breast carcinomas, especially when lesions are non-palpable and subcentimetric, due to the limited spatial resolution of the device $(11,12)$.

At present, this limitation may be overcome with the employment of high-resolution, small-field of view gamma-cameras specifically designed for the imaging of the breasts (13). This imaging procedure, namely breast specific gamma imaging (BSGI) or molecular breast imaging (MBI), has been demonstrated to be more sensitive than conventional breast scintigraphy, especially in detecting small size primary breast carcinomas, while maintaining the same high specificity (14).

In clinical practice, MBI has been established to be able to play an important complementary role to mammography in the diagnosis of primary breast cancer, especially in indeterminate or inconclusive mammography findings, increasing mammography sensitivity in dense breast and in multifocal/multicentric disease (15-18). MBI has also demonstrated similar high sensitivity values as mammography in the detection of ductal carcinoma in situ, irrespective of histological subtype, and with a scintigraphic pattern of uptake that correlated well with mammography findings (19).

Moreover, in some studies, MBI has provided a better preoperative local staging than mammography, altering surgical management and guiding the surgeon to a more appropriate surgical treatment $(17,18,20)$.

MBI acquired with the newly developed dual-headed cadmium-zinc-telluride (CdZnTe or CZT) devices at a reduced radiation dose, has also proved to be able to increase the sensitivity of mammography in screening women with mammographically dense breast, yielding a supplemental cancer detection rate of 8.8/1,000 screened women (21).

In the present study we retrospectively assessed the usefulness of MBI in predicting complete tumor response to treatment and residual tumor extent following neoadjuvant therapy in a consecutive series of patients with large or locally advanced primary breast cancer using surgical histopathological findings as the gold standard.

\section{Materials and methods}

Patients. We retrospectively reviewed a consecutive series of 43 female patients with large or locally advanced primary breast cancer scheduled to receive neoadjuvant therapy who preoperatively, at the end of their neoadjuvant treatment regimen, were submitted to MBI. All patients were followed by the same team of oncologists who at baseline, before starting neoadjuvant therapy, submitted patients to local (breast and axillary) clinical examination, conventional diagnostic imaging procedures (including mammography and breast ultrasonography) and tumor biopsy to assess histological type, nuclear grade and the level of expression of estrogen receptor (ER), progesterone receptor (PR) and HER2/neu receptor. Ki-67 (MIB1) index of tumor proliferation was also assessed on biopsy samples. Tumors were classified in three molecular subtypes according to their receptor status: luminal $\left(\mathrm{ER}^{+}\right.$or $\left.\mathrm{PR}^{+}\right)$, HER2 (ER', $\mathrm{PR}^{-}$and HER2/ne $\left.{ }^{+}\right)$, and triple-negative (ER', $\mathrm{PR}^{-}$and $\mathrm{HER} 2 /$ ne $\left.^{-}\right)$. MBI was performed also at baseline, before starting the neoadjuvant treatment. All investigations were performed in a University Hospital setting, as part of the clinical care of breast cancer patients.

According to clinical, imaging and biopsy data, 35/43 patients were scheduled to receive neoadjuvant chemotherapy: a cisplatin-based therapy in 19 cases and an anthracycline-based therapy in 26 cases (plus paclitaxel in 10/26 cases); $6 / 35$ patients were also treated with trastuzumab. The remaining $8 / 43$ patients, all elderly in the post-menopausal phase and with ER-positive tumors, were submitted to anti-hormonal treatment with the aromatase inhibitor exemestane.

At the end of the neoadjuvant treatment, all patients underwent breast surgery that was planned and performed by the same team of surgeons. A total of 30/43 patients underwent a mastectomy and 13/43 patients had breast-conserving surgery (BCS). Axillary lymph node dissection (ALND) was also performed in 38/43 cases, while ALND was avoided in the remaining 5/43 patients, all elderly, submitted to anti-hormonal therapy and with clinically negative axillary lymph nodes.

Patients with concomitant distant metastases ascertained at standard staging diagnostic procedures (chest computed tomography, bone scan and abdomen ultrasound or computed tomography) and patients scheduled for concomitant radiotherapy were excluded from this study.

The clinicopathological characteristics of the 43 patients enrolled in the study are listed in Table I. The baseline clinical tumor $(\mathrm{T})$ and lymph node $(\mathrm{N})$ classification was performed according to the American Joint Committee on Cancer Criteria.

Breast scintigraphy. MBI was performed $10 \mathrm{~min}$ after the intravenous injection of $740 \mathrm{MBq}$ of ${ }^{99 \mathrm{~m}} \mathrm{Tc}-$ tetrofosmin (Myoview; GE Healthcare, Oslo, Norway) in the arm contralateral to the affected breast. Radiolabelling and quality control procedures of the radiotracer were carried out according to the manufacturer's instructions. Labelling efficiency was always $>95 \%$. Breast images were acquired using a high-resolution, solid-state dedicated breast camera that is composed of a semiconductor detector with a pixelated array of CZT coupled to an array of amplifiers, the signals from which are conveyed on an electronic readout board. The system is modelled to have an intrinsic spatial resolution of $1.6 \mathrm{~mm}$ and an energy resolution of $<5 \%$. In all cases the breast images were acquired in both craniocaudal and mediolateral oblique projections (600 sec/view), using a 128x128 matrix size, with mild breast compression during acquisition. Additional projections could be acquired when necessary (such as breasts bigger than the field of view and areas of increased uptake at the border of the field of view).Written informed patient consent was always obtained before the scintigraphy.

Data analysis. MBI images were independently evaluated by two experienced nuclear medicine physicians (AS and GM) 
Table I. Patient characteristics.

\begin{tabular}{|c|c|}
\hline Characteristics & No. of patients \\
\hline \multicolumn{2}{|l|}{ Age at diagnosis (years) } \\
\hline$<50$ & 10 \\
\hline$\geq 50$ & 33 \\
\hline \multicolumn{2}{|l|}{ Menopausal status } \\
\hline Pre-menopausal & 17 \\
\hline Post-menopausal & 26 \\
\hline \multicolumn{2}{|l|}{ Clinical TN stage } \\
\hline IIA & 24 \\
\hline IIB & 5 \\
\hline IIIA & 7 \\
\hline IIIB & 7 \\
\hline \multicolumn{2}{|l|}{ Tumor histology } \\
\hline Invasive ductal & 36 \\
\hline Invasive lobular & 3 \\
\hline Invasive mucinous & 1 \\
\hline Tubular & 1 \\
\hline Epithelial & 1 \\
\hline Metaplastic & 1 \\
\hline \multicolumn{2}{|l|}{ Molecular subtype } \\
\hline Luminal & 29 \\
\hline HER2 & 6 \\
\hline Triple-negative & 8 \\
\hline \multicolumn{2}{|l|}{ Neoadjuvant treatment } \\
\hline Chemotherapy & 29 \\
\hline Chemotherapy plus trastuzumab & 6 \\
\hline Anti-hormonal & 8 \\
\hline
\end{tabular}

who were blinded to the final histopathological findings that represented the gold standard. Disagreements were resolved by consensus.

MBI images were considered negative for residual tumors in the absence of detectable uptake on the post-therapy scan while they were considered positive in the presence of one or more areas (either focal or patchy) of increased uptake (mild, moderate or intense) at the level of the primary tumor site well distinct from background breast activity. In presence of a focal area of increased uptake with well-delineated contours, lesion size was assessed manually using an image function present in the Xeleris 2.0 Workstation System (GE Healthcare) available in our Nuclear Medicine Unit. The size was determined according to the largest diameter measured on mediolateral oblique and craniocaudal images.

MBI results were related to the histopathological findings obtained from surgical samples.

Histopathology. Histopathological analysis was performed according to standard procedures. A macroscopic analysis was performed with the size of residual tumors determined according to the largest diameter. According to the number of tumor foci, the carcinomas were classified as unifocal (only one focus) or multifocal/multicentric (two or more tumor foci within a single breast or within different quadrants of the same breast). Breast surgical specimens were then fixed in $10 \%$ buffered formalin and stained with haematoxylin and eosin (H\&E). A further histochemical analysis was performed with microscopic evaluation.

The pathological response to neoadjuvant therapy was classified as follows: complete response, disappearance in surgical specimens of target lesions observed at the baseline mammography; partial response, at least a $30 \%$ decrease in the diameter of target lesions, adopting as the reference the baseline mammographic diameter; progressive disease, at least a $20 \%$ increase in the diameter of target lesions; stable disease, neither sufficient shrinkage to qualify for partial response nor sufficient increase to qualify for progressive disease.

Statistical analysis. Post-therapy images obtained using MBI were considered as positive, negative, false-positive or false-negative for residual breast tumors considering the histopathological findings obtained at surgery as the 'gold standard'. Sensitivity, specificity, positive predictive value (PPV), negative predictive value (NPV) and accuracy were then calculated. The Spearman's correlation coefficient was calculated to analyze the residual tumor size with MBI and histopathological examination.

\section{Results}

Seven out of 43 patients (16.3\%), 6 following chemo- and 1 following anti-hormonal therapy, exhibited a pathological complete response, while the remaining 36/43 patients (83.7\%), 29 following chemo- and 7 following anti-hormonal therapy, were classified as partial responders (28 cases) or with stable/progressive disease ( 8 cases) and had residual tumors at surgery. The latter was unifocal in $27 / 36$ cases and multicentric/multifocal in the remaining 9/36 cases.

The 27 unifocal residual tumors included 25 invasive ductal carcinomas, 1 mucinous and 1 metaplastic carcinoma, and ranged in size from 1.0 to $7.0 \mathrm{~cm}$, with a mean lesion size of $2.411 \pm 2.361 \mathrm{~cm}$ at histopathological analysis.

The histopathological findings regarding the 9 patients with multifocal/multicentric residual disease, including tumor histology, number of lesions and lesion size, are listed in Table II.

From the images obtained using MBI, 7 patients were negative for residual tumors with pathological complete response, demonstrating a complete disappearance of radiotracer uptake on the post-therapy scan (one of these cases is illustrated in Fig. 1), while 34/36 patients were positive for residual tumors. Sensitivity, specificity, PPV, NPV and accuracy values are reported in Table III.

MBI detected 26/27 unifocal residues and failed in the detection of one $2.5-\mathrm{cm}$ invasive ductal carcinoma deeply located in the internal mammary quadrant of the left breast, excluded from the field of view during acquisition. The 26 positive unifocal residual tumors appeared in MBI as focal areas of intense increased uptake of the radiotracer with well-delineated contours, with a mean lesion size of $2.361 \pm 1.374 \mathrm{~cm}$. Scatter diagram and the correlation of tumor size between MBI and histopathology is shown in Fig. 2. One of the patients with unifocal residual tumors correctly visualized and assessed in 
Table II. Histopathological findings regarding the 9 patients with multifocal/multicentric residual tumors.

Tumor histology (no. of patients)

No. of lesions and lesion size

Invasive ductal plus invasive lobular $(\mathrm{n}=1)$

Invasive ductal $(\mathrm{n}=1)$

Invasive lobular $(\mathrm{n}=3)$

Poor differentiated DCSIS and small invasive carcinoma $(n=1)$

Epithelial carcinoma $(\mathrm{n}=1)$

Tubular carcinoma $(n=1)$

Invasive ductal mixed with DCIS $(n=1)$
1 focus $5 \mathrm{~cm}$

1 focus $1.5 \mathrm{~cm}$

2 foci 1.5 and $1.2 \mathrm{~cm}$, respectively

Diffuse microscopic foci (1 case)

Multiple microscopic foci scattered in a $4-\mathrm{cm}$ area (1 case)

Multiple microscopic foci scattered in a $1.5-\mathrm{cm}$ area (1 case)

Rare microscopic foci scattered in a fibrotic $3-\mathrm{cm}$ area

Multiple microscopic foci scattered in a fibrotic 1-cm area

Multifocal microscopic foci with a total extension of $4 \mathrm{~cm}$

Multiple microscopic foci scattered in a fibrotic $1-\mathrm{cm}$ area
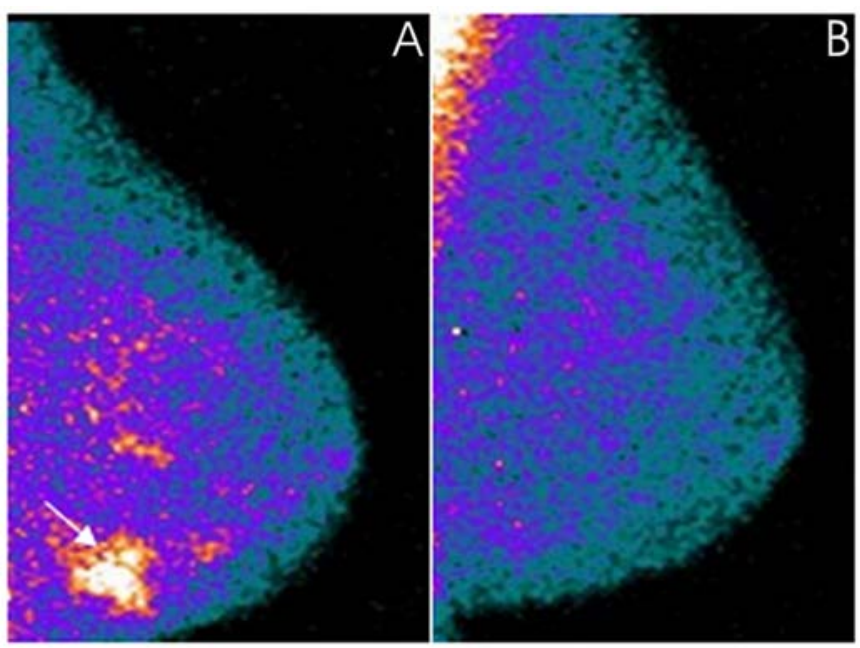

Figure 1. A 71-year-old patient with locally advanced invasive ductal carcinoma (clinical stage at baseline: IIA) located in the external lower quadrant of the left breast that demonstrated pathological complete remission after neoadjuvant chemotherapy. MBI, in a mediolateral oblique view, was positive in the (A) pre-therapy study at the level of the primary tumor (arrow) while the radiotracer uptake was no longer evident in the (B) post-therapy study. MBI, molecular breast imaging.

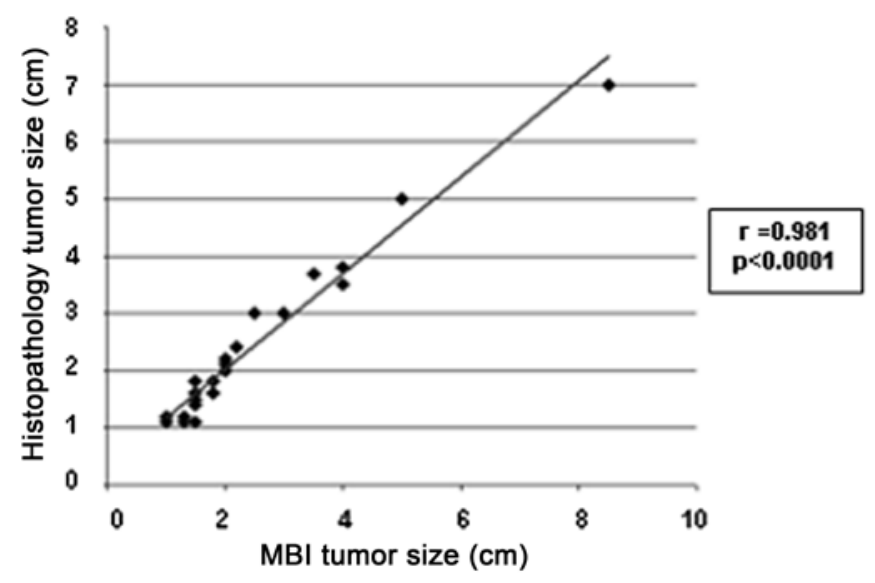

Figure 2. Scatter diagram of the correlation between tumor size (in $\mathrm{cm}$ ) obtained with MBI (x-axis) and histopathology (y-axis) regarding the 26 positive unifocal residual tumors as determined with MBI. MBI, molecular breast imaging.
Table III. MBI results in residual breast cancer detection after neoadjuvant therapy in the 43 patients enrolled in the study, 7 without and 36 with residual disease after neoadjuvant therapy.

\begin{tabular}{lc}
\hline Parameters & Data \\
\hline Negative & 7 \\
False-positive & 0 \\
Positive & 34 \\
False-negative & 2 \\
Sensitivity & $94.4 \%(34 / 36)$ \\
Specificity & $100 \%(7 / 7)$ \\
Accuracy & $95.3 \%(41 / 43)$ \\
Positive predictive value & $100 \%(34 / 34)$ \\
Negative predictive value & $77.8 \%(7 / 9)$
\end{tabular}

MBI, molecular breast imaging.

size with MBI is illustrated in Fig. 3. MBI was in agreement with surgery in identifying only one lesion in $25 / 26$ positive cases; in the remaining patients, MBI correctly identified and assessed the size of the residual tumor ascertained at surgery (a 2-cm invasive ductal carcinoma) but overestimated disease extent also identifying two small focal areas of increased uptake surrounding the residual tumor.

Moreover, MBI was positive in $8 / 9$ multicentric/multifical residual tumors and false-negative in one patient with rare microscopic foci of epithelial carcinoma (luminal subtype, MIB1: $15 \%$ ) scattered in a fibrotic area $1-\mathrm{cm}$ large. MBI findings were concordant with histopathological analysis in assessing the number of lesions and lesion size in the patient with one large focus $(5 \mathrm{~cm})$ of invasive ductal carcinoma and one smaller focus $(1.5 \mathrm{~cm})$ of invasive lobular carcinoma, while it overestimated the number of lesions in the other patient with 2 surgically proven foci of invasive ductal carcinoma. In the remaining $6 / 8$ positive patients with microscopic multifocal/multicentric tumoral foci, MBI revealed patchy areas with irregular borders of radiotracer uptake of mild to 


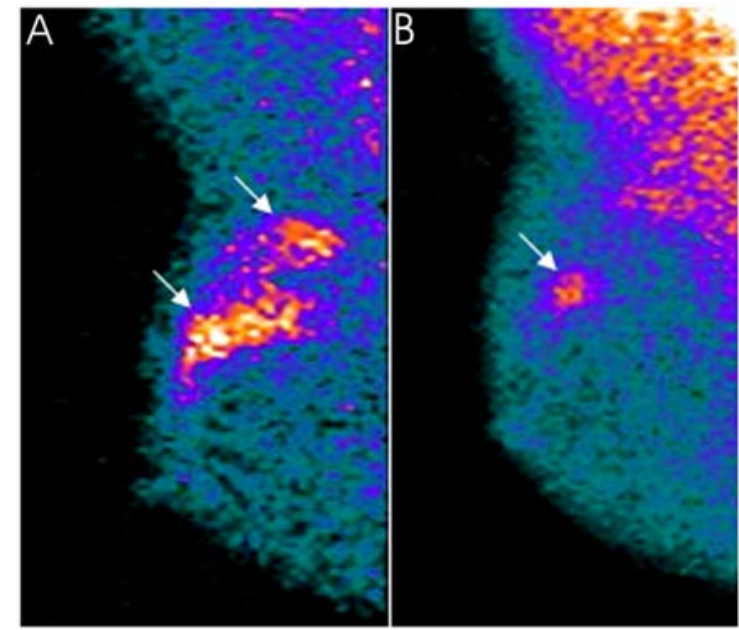

Figure 3. A 47-year-old patient with locally advanced invasive ductal carcinoma (clinical stage at baseline: IIA) located in the external upper quadrant of the right breast clearly evident (arrows) in MBI in a the mediolateral oblique view in the (A) pre-therapy study. The patient was classified as a partial responder after neoadjuvant chemotherapy. At surgery, a unifocal residual tumor $1.5-\mathrm{cm}$ large was ascertained in a (B) post-therapy MBI scan as a focal area of increased uptake (arrow) whose size corresponded exactly to the histopathological result. MBI, molecular breast imaging.

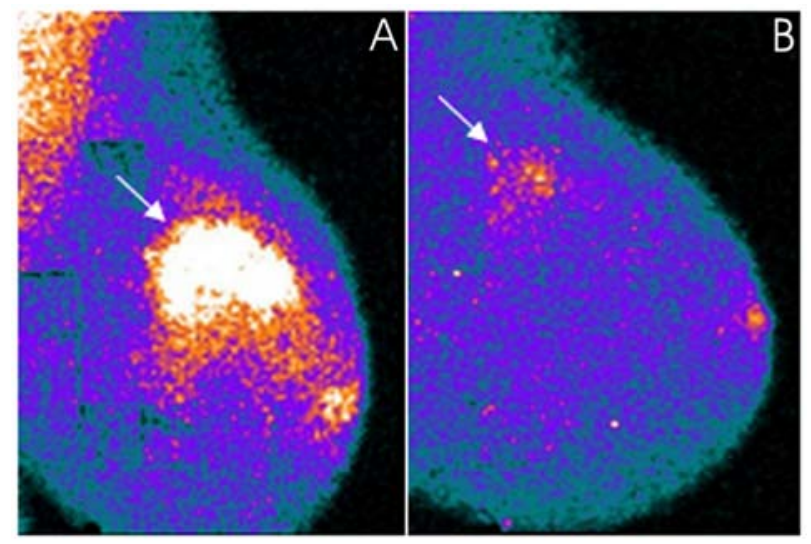

Figure 4. A 45-year-old patient with locally advanced invasive ductal carcinoma (clinical stage at baseline: IIB) located in the external upper quadrant of the left breast clearly evident with MBI (arrow) in a mediolateral oblique view in the (A) pre-therapy study. The patient was classified as a partial responder after neoadjuvant chemotherapy. Multiple microscopic foci of invasive ductal carcinoma mixed with DCIS scattered in a fibrotic area $3-\mathrm{cm}$ large were found at surgery. Residual disease was evident in an (B) MBI post-therapy scan as a patchy area with irregular borders of mild increased radiotracer uptake (arrow). The largest diameter measured with MBI concurred with histopathological disease extent. MBI, molecular breast imaging.

moderate intensity at the level of the residual disease. The largest diameter of these areas was in agrrement with histopathological analysis in 4/6 cases (one of these cases is shown in Fig. 4), while in the remaining 2/6 cases, one with a multicentric invasive lobular carcinoma (luminal subtype, MIB1: $3 \%$ ) and one with rare microscopic foci of tubular carcinoma (luminal subtype, MIB1: $8 \%$ ), both scattered in a fibrotic area of $4 \mathrm{~cm}$ in size, tumor extent was underestimated with MBI by 1 and by $1.5 \mathrm{~cm}$, respectively.

Collectively, excluding the 2 false-negative patients and the 4 overestimated or underestimated cases, MBI findings were concordant with histopathological data in correctly predicting complete response to treatment and residual tumor extent in 37 of the 43 patients $(86 \%)$ enrolled in the study.

\section{Discussion}

The recent development of high-resolution, small-field of view dedicated breast cameras has opened a new era for the radioisotopic imaging of the breasts with the technetium-labelled cationic lipophilic radiotracers sestamibi and tetrofosmin.

The advantages offered by these new devices in respect to conventional general-purpose gamma cameras are numerous. First of all, they offer the possibility of focalising the imaging to the breast that is placed directly on the camera face, excluding from the field of view the nearby organs that physiologically accumulate on the radiotracer (i.e., liver and heart); this permits the acquirement of images in projections similar to those of mammography facilitating the correlation between the two types of images (22).

Moreover, dedicated breast cameras are characterized by a significantly higher intrinsic spatial resolution, especially when solid-state semiconductor CZT devices are employed, increasing the capacity of detecting subcentimetric lesions. Using the more recently available dual-headed CZT breast cameras, that are characterized by optimized collimation and a wide energy window, sensitivity can be further increased and the administered dose of radiotracer may be reduced minimizing radiation exposure to patients $(23,24)$.

Due to the aforementioned favourable characteristics, MBI is now considered the radioisotopic imaging method of choice in the diagnosis of primary breast cancer, replacing conventional planar scintimammography.

The possible role of MBI in monitoring the local response of breast cancer to neoadjuvant therapy is still under investigation. At present, only a few studies have been reported in literature focusing on this matter. Moreover, these studies are not homogeneous with regard to the device employed and study design and have presented conflicting results.

In a pilot study carried out on a series of 20 patients, 14 of whom exhibited residual tumors after therapy, measurements of tumor size by MBI and decrements of T/B (tumor-to-background) ratios in images acquired before and at completion of neoadjuvant therapy demonstrated a limited predictive value regarding the pathological extent of residual disease (25).

Other researchers evaluated the accuracy of preoperative MBI in assessing residual tumor presence and residual tumor size in comparison with MRI in a series of 122 breast cancer patients treated with neoadjuvant chemotherapy. The sensitivity and the specificity of MBI for residual tumor detection were 74.0 and $72.2 \%$, respectively, comparable to those of MRI, 81.7 and $72.2 \%$ (26). In this series, the assessment of residual tumor extent appeared to depend on the molecular subtype, the residual tumor size being significantly underestimated by MBI in the luminal subtype and by MRI in both luminal and HER2 subtypes. Both procedures provided accurate tumor size measurements in the triple-negative subtype (26). The higher rate of underestimation in the luminal and/or HER2 subtypes by MBI was explained by the researchers and attributed to low-residual cellularity (26).

The ability of MBI to accurately assess residual disease was demonstrated to be associated with the molecular subtype 
also in a more recent study carried out on 51 patients, in which the highest accuracy was observed in the triple-negative and HER2/neu positive subtypes (27).

In the present study, we retrospectively assessed the usefulness of MBI, performed before and at the completion of chemo- or anti-hormonal neoadjuvant therapy, in predicting complete tumor response to treatment and residual tumor extent in a consecutive series of 43 patients, considering histopathological analysis on surgical samples as the gold standard.

In our series, a pathological complete response, with total disappearance of the primary breast tumor, was observed in $16.3 \%$ of the cases, while tumor residues, variable in size from a few millimetres to several centimetres, were ascertained at surgery in the remaining $83.7 \%$ of cases.

MBI proved to be a very high-specific imaging method, resulting in accurate negative images in all patients without residual disease, thus confirming the usefulness of radioisotopic procedures with cationic lipophilic radiotracers in differentiating tumor residues from areas of fibrotic tissue replacement inducted by neoadjuvant treatments, as previously observed in breast cancer and in other types of carcinomas (28-30). The absence of false-positive results in our series may be explained by the tetrofosmin cellular uptake mechanism, the radiotracer accumulating only in viable cells, but not in fibrotic tissues.

MBI also demonstrated a high sensitivity, detecting residual disease in $94.4 \%$ of cases. We observed only 2 false-negative cases; one of these was probably related to the tumor site, since the lesion was deeply located in the internal mammary quadrant and not included in the field of view of the detector, while the second case could be explained by the small size of the residual tumor foci that were microscopic, besides being rare and scattered in a relatively small $(1 \mathrm{~cm})$ fibrotic area. The technical limitation of MBI in visualizing breast tumors located close to the chest wall is well recognized (18); it is also known that the sensitivity of the method is partially related to lesion size (18). However, in this series, MBI demonstrated a high performance also in the identification of microscopic and/or diffuse residual disease, confirming our previous preliminary data (31). Thus, it is likely that not only the size of neoplastic foci, especially when under the spatial resolution of the detector, but also other factors, specifically related to tumor biology (i.e., histological subtype, slow growth rate and low cellularity), can affect the identification of residual breast tumors.

Moreover, in the present study, the extent of residual disease identified with MBI correlated well with histopathological analysis, particularly when residual tumors were unifocal.

Collectively, MBI data concurred with histopathological analysis from surgical samples in a high percentage of cases (86\%), thus suggesting that the procedure may represent a useful diagnostic imaging test to assess the response to neoadjuvant chemo- or anti-hormonal therapy in breast cancer patients and in assessing residual tumor extent, guiding the surgeon in planning the most appropriate surgical treatment of the involved breast. In the era of BCS, the knowledge of the extent of residual disease is of extreme importance, the goal being the complete excision of residues with a clear margin excision.

In our series, disease extent was underestimated in 2 patients with microscopic foci of invasive carcinoma, lobular in one case and tubular in the other, scattered in a fibrotic area. Both underestimated cases belonged to the luminal subtype and had a low cellularity. Thus, it is possible that molecular subtype and cellularity degree may affect tumor size assessment in MBI, as also hypothesized by other researchers $(26,27)$.

In the present study, MBI was used at the end of the therapeutic cycles, but in the future, it would be interesting to perform MBI also during therapy in order to identify early in the treatment method non-responder patients who could benefit from a change of the therapeutic regimen. This approach has been tested by some researchers in a series of 19 patients in whom MBI was performed before (baseline), at 3-5 weeks after onset, and after completion of therapy (32). Changes in T/B ratios on MBI images performed at 3-5 weeks following initiation of therapy were accurate in predicting the presence or absence of residual disease at therapy completion (32).

An important limitation of MBI remains its inability in detecting axillary lymph node metastases. It is well known that axillary lymph node status after neoadjuvant therapy represents another important predictor of disease-free and overall survival in breast cancer patients. Following neoadjuvant therapies, ALND is thus generally performed for nodal staging at the time of breast surgery, although a potential role has been recently hypothesized for the radioguided sentinel node biopsy in selected cases.

Other radioisotopic procedures, such as single-photon emission computed tomography (SPECT), preferably with pinhole collimator (pinhole-SPECT), and SPECT combined with computed tomography (SPECT/CT), have proved more suitable than both conventional planar scintimammography and MBI in axillary lymph node metastasis detection, facilitating the identification of non-palpable and deeply located axillary lymph node metastases (33-37). An acquisition protocol that includes an MBI study followed by a SPECT scan of the axillary regions, could represent a useful diagnostic option to obtain information regarding both residual breast disease and axillary lymph node status concurrently, thus optimizing the surgical approach not only at the level of the breast but also at the level of the axilla.

The present study has some limitations. It is a retrospective single-institution study that involves a limited number of cases. Thus, larger prospective multi-centre trials are warranted to further determine the utility of MBI in patients with large or locally advanced primary breast cancer following neoadjuvant therapy and its effect on patient management.

In conclusion, MBI proved to be a highly accurate diagnostic tool in predicting complete tumor response to treatment and residual tumor extent following neoadjuvant chemotherapy or anti-hormonal therapy in patients with large or locally advanced primary breast cancer, concurring with surgical histopathological findings in $86 \%$ of overall cases. A positive result was always associated with the presence of residual disease and MBI tumor size was strongly correlated with histopathological analysis mainly in unifocal tumors.

Our data revealed a wider application of this procedure in the preoperative management of breast cancer patients scheduled to receive surgery following neoadjuvant therapy, to guide the surgeon to the most appropriate breast surgical 
treatment. However, these data need to be confirmed in larger prospective studies.

\section{Acknowledgements}

Not applicable.

\section{Funding}

No funding was received.

\section{Availability of data and materials}

The analyzed data sets generated during the study are available from the corresponding author upon reasonable request.

\section{Authors' contributions}

GM and AS designed the research. SN mainly did the research. SG, IG and AF helped to collect the data from the patients. SG also performed statistical analysis. GR and PAS analyzed the data. All authors read and approved the manuscript and agree to be accountable for all aspects of the research in ensuring that the accuracy or integrity of any part of the study are appropriately investigated and resolved.

\section{Ethics approval and consent to participate}

This retrospective study was carried out in accordance with the ethical standards of the institutional research committee and with the 1964 Helsinki declaration and its later amendments or ethical standards. The requirement to obtain informed consent was waived as this was a retrospective study. All patient data were treated in accordance with the local privacy regulations.

\section{Consent for publication}

Not applicable.

\section{Competing interests}

The authors declare that they have no competing interests.

\section{References}

1. Untch M, Konecny GE, Paepke S and von Minckwitz G: Current and future role of neoadjuvant therapy for breast cancer. Breast 23: 526-537, 2014.

2. Newman LA: Management of patients with locally advanced breast cancer. Curr Oncol Rep 6: 53-61, 2004.

3. Kong X, Moran MS,Zhang N, Haffty B and Yang Q: Meta-analysis confirms achieving pathological complete response after neoadjuvant chemotherapy predicts favourable prognosis for breast cancer patients. Eur J Cancer 47: 2084-2090, 2011.

4. Chagpar AB, Middleton LP, Sahin AA, Dempsey P, Buzdar AU, Mirza AN, Ames FC, Babiera GV, Feig BW, Hunt KK, et al: Accuracy of physical examination, ultrasonography, and mammography in predicting residual pathologic tumor size in patients treated with neoadjuvant chemotherapy. Ann Surg 243: 257-264, 2006.

5. Spanu A, Farris A, Schillaci O, Chessa F, Solinas ME, Falchi A, Madeddu G, Nuvoli S and Madeddu G: The usefulness of ${ }^{99 \mathrm{~m}} \mathrm{Tc}$ tetrofosmin scintigraphy in patients with breast cancer recurrences. Nucl Med Commun 24: 145-154, 2003.
6. Spanu A, Schillaci O and Madeddu G: ${ }^{99 \mathrm{~m}} \mathrm{Tc}$ labelled cationic lipophilic complexes in malignant and benign tumors: The role of SPET and pinhole-SPET in breast cancer, differentiated thyroid carcinoma and hyperparathyroidism. Q J Nucl Med Mol Imaging 49: 145-169, 2005.

7. Schillaci O, Spanu A, Danieli R and Madeddu G: Molecular breast imaging with gamma emitters. Q J Nucl Med Mol Imaging 57: 340-351, 2013.

8. Arbab AS, Koizumi K, Toyama K, Arai T and Araki T: Ion transport systems in the uptake of ${ }^{99} \mathrm{Tcm}$-tetrofosmin, ${ }^{99} \mathrm{Tcm}-\mathrm{MIBI}$ and 201Tl in a tumour cell line. Nucl Med Commun 18: 235-240, 1997.

9. Bernard BF, Krenning EP, Breeman WA, Ensing G, Benjamins H, Bakker WH, Visser TJ and de Jong M: ${ }^{99 \mathrm{~m} T c-M I B I},{ }^{99 \mathrm{~m} T c-t e t r o-}$ fosmin and ${ }^{99 \mathrm{~m}} \mathrm{Tc}-\mathrm{Q} 12$ in vitro and in vivo. Nucl Med Biol 25: 233-240, 1998.

10. Arbab AS, Koizumi K, Toyama K, Arai T and Araki T: Technetium-99m-tetrofosmin, technetium-99m-MIBI and thallium-201 uptake in rat myocardial cells. J Nucl Med 39: 266-271, 1998.

11. Spanu A, Dettori G, Nuvoli S, Porcu A, Falchi A, Cottu P, Solinas ME, Scanu AM, Chessa F and Madeddu G: (99)mTc-tetrofosmin SPET in the detection of both primary breast cancer and axillary lymph node metastasis. Eur J Nucl Med 28: 1781-1794, 2001.

12. Spanu A, Schillaci O, Meloni GB, Porcu A, Cottu P, Nuvoli S, Falchi A, Chessa F, Solinas ME and Madeddu G: The usefulness of ${ }^{99 \mathrm{~m}} \mathrm{Tc}$-tetrofosmin SPECT scintimammography in the detection of small size primary breast carcinomas. Int J Oncol 21: 831-840, 2002.

13. Goldsmith SJ, Parsons W, Guiberteau MJ, Stern LH, Lanzkowsky L, Weigert J, Heston TF, Jones E, Buscombe J and Stabin MG; Society of Nuclear Medicine: SNM practice guideline for breast scintigraphy with breast-specific gamma-cameras 1.0 . J Nucl Med Technol 38: 219-224, 2010.

14. Brem RF, Schoonjans JM, Kieper DA, Majewski S, Goodman S and Civelek C: High-resolution scintimammography: A pilot study. J Nucl Med 43: 909-915, 2002.

15. Spanu A, Cottu P, Manca A, Chessa F, Sanna D and Madeddu G: Scintimammography with dedicated breast camera in unifocal and multifocal/multicentric primary breast cancer detection: A comparative study with SPECT. Int J Oncol 31: 369-377, 2007.

16. Spanu A, Chessa F, Meloni GB, Sanna D, Cottu P, Manca A, Nuvoli $S$ and Madeddu G: The role of planar scintimammography with high-resolution dedicated breast camera in the diagnosis of primary breast cancer. Clin Nucl Med 33: 739-742, 2008.

17. Spanu A, Sanna D, Chessa F, Manca A, Cottu P, Fancellu A, Nuvoli S and Madeddu G: The clinical impact of breast scintigraphy acquired with a breast specific $\gamma$-camera (BSGC) in the diagnosis of breast cancer: Incremental value versus mammography. Int J Oncol 41: 483-489, 2012.

18. Sun Y, Wei W, Yang HW and Liu JL: Clinical usefulness of breast-specific gamma imaging as an adjunct modality to mammography for diagnosis of breast cancer: A systemic review and meta-analysis. Eur J Nucl Med Mol Imaging 40: 450-463, 2013.

19. Spanu A, Sanna D, Chessa F, Cottu P, Manca A and Madeddu G: Breast scintigraphy with breast-specific $\gamma$-camera in the detection of ductal carcinoma in situ: A correlation with mammography and histologic subtype. J Nucl Med 53: 1528-1533, 2012.

20. Edwards C, Williams S, McSwain AP, Damle S, Rapelyea JA, Downs K, Torrente J, Sambamurty A, Brem RF and Teal CB: Breast-specific gamma imaging influences surgical management in patients with breast cancer. Breast J 19: 512-519, 2013.

21. Rhodes DJ, Hruska CB, Conners AL, Tortorelli CL, Maxwell RW, Jones KN, Toledano AY and O'Connor MK: Journal club: Molecular breast imaging at reduced radiation dose for supplemental screening in mammographically dense breasts. AJR Am J Roentgenol 204: 241-251, 2015.

22. Hsu DF, Freese DL and Levin CS: Breast-Dedicated Radionuclide Imaging Systems. J Nucl Med 57 (Suppl 1): 40S-45S, 2016.

23. Hruska CB, Phillips SW, Whaley DH, Rhodes DJ and O'Connor MK: Molecular breast imaging: Use of a dual-head dedicated gamma camera to detect small breast tumors. AJR Am J Roentgenol 191: 1805-1815, 2008.

24. Hruska CB, Weinmann AL, Tello Skjerseth CM, Wagenaar EM, Conners AL, Tortorelli CL, Maxwell RW, Rhodes DJ and O'Connor MK: Proof of concept for low-dose molecular breast imaging with a dual-head CZT gamma camera. Part II. Evaluation in patients. Med Phys 39: 3476-3483, 2012. 
25. Wahner-Roedler DL, Boughey JC, Hruska CB, Chen B Rhodes DJ, Tortorelli CL, Maxwell RW, Cha SS and $\mathrm{O}^{\prime}$ Connor MK: The use of molecular breast imaging to assess response in women undergoing neoadjuvant therapy for breast cancer: A pilot study. Clin Nucl Med 37: 344-350, 2012

26. Lee HS, Ko BS, Ahn SH, Son BH, Lee JW, Kim HJ, Yu JH, Kim SB, Jung KH, Ahn JH, et al: Diagnostic performance of breast-specific gamma imaging in the assessment of residual tumor after neoadjuvant chemotherapy in breast cancer patients. Breast Cancer Res Treat 145: 91-100, 2014.

27. Menes TS, Golan O, Vainer G, Lerman H, Schneebaum S, Klausner J and Even-Sapir E: Assessment of residual disease with molecular breast imaging in patients undergoing neoadjuvant therapy: Association with molecular subtypes. Clin Breast Cancer 16: 389-395, 2016.

28. Spanu A, Ginesu F, Pirina P, Solinas ME, Schillaci O, Farris A Chessa F, Madeddu G, Marongiu P, Falchi A, et al: The usefulness of ${ }^{99 \mathrm{~m} T c}$-tetrofosmin SPECT in the detection of intrathoracic malignant lesions. Int J Oncol 22: 639-649, 2003.

29. Schillaci O, Spanu A and Madeddu G: ${ }^{[99 \mathrm{~m}}$ Tc] $]$ sestamibi and $\left.{ }^{99 \mathrm{~m}} \mathrm{Tc}\right]$ tetrofosmin in oncology: SPET and fusion imaging in lung cancer, malignant lymphomas and brain tumors. Q J Nucl Med Mol Imaging 49: 133-144, 2005.

30. Spanu A, Sanna D, Chessa F, Farris A, Nuvoli S and Madeddu G: The usefulness of Tc-99m-tetrofosmin SPECT/CT in the detection of residual tumors and axillary lymph node metastases in breast cancer patients following neoadjuvant therapy. Clin Nucl Med 36: 997-1002, 2011.

31. Spanu A, Farris A, Chessa F, Sanna D, Pittalis M, Manca A and Madeddu G: Planar scintimammography and SPECT in neoadjuvant chemo or hormonotherapy response evaluation in locally advanced primary breast cancer. Int J Oncol 32: 1275-1283, 2008.

32. Mitchell D, Hruska CB, Boughey JC, Wahner-Roedler DL, Jones KN, Tortorelli C, Conners AL and O'Connor MK: ${ }^{99 \mathrm{~m}}$ Tc-sestamibi using a direct conversion molecular breast imaging system to assess tumor response to neoadjuvant chemotherapy in women with locally advanced breast cancer. Clin Nucl Med 38: 949-956, 2013
33. Schillaci O, Scopinaro F, Spanu A, Donnetti M, Danieli R, Di Luzio E, Madeddu G and David V: Detection of axillary lymph node metastases in breast cancer with Tc-99m tetrofosmin scintigraphy. Int J Oncol 20: 483-487, 2002.

34. Spanu A, Tanda F, Dettori G, Manca A, Chessa F,Porcu A,Falchi A, Nuvoli S and Madeddu G: The role of $(99 \mathrm{~m}) \mathrm{Tc}$-tetrofosmin pinhole-SPECT in breast cancer non palpable axillary lymph node metastases detection. Q J Nucl Med 47: 116-128, 2003.

35. Madeddu G and Spanu A: Use of tomographic nuclear medicine procedures, SPECT and pinhole SPECT, with cationic lipophilic radiotracers for the evaluation of axillary lymph node status in breast cancer patients. Eur J Nucl Med Mol Imaging 31 (Suppl 1): S23-S34, 2004.

36. Spanu A, Chessa F, Sanna D, Cottu P, Manca A, Nuvoli S and Madeddu G: Breast cancer axillary lymph node metastasis detection by a high-resolution dedicated breast camera: A comparative study with SPECT and pinhole SPECT. Cancer Biother Radiopharm 22: 799-811, 2007.

37. Spanu A, Chessa F, Sanna D, Cottu P, Manca A, Nuvoli S and Madeddu G: Scintimammography with a high resolution dedicated breast camera in comparison with SPECT/CT in primary breast cancer detection. Q J Nucl Med Mol Imaging 53: 271-280, 2009.

This work is licensed under a Creative Commons Attribution-NonCommercial-NoDerivatives 4.0 International (CC BY-NC-ND 4.0) License. 